\title{
[gw22-e0687] COMMON GENETIC VARIANTS ON CHROMOSOME 9P21 ARE ASSOCIATED WITH THE RISK AND SEVERITY OF CORONARY ARTERY DISEASE IN TURKISH POPULATION
}

Huseyin Altug Cakmak¹, Burcu Bayoglu², Erkan Yildirim', Eser Durmaz', Bilgehan Karadag', Gunay Can², Mujgan Censgiz ${ }^{2}$, Vural Ali Vural', Husniye Yukse ${ }^{1}{ }^{1} /$ stanbul University, Cerrahpasa Medical Faculty, Department Of Cardiology, Istanbul, Turkey; ${ }^{2}$ Istanbul University, Cerrahpasa Medical Faculty, Department Of Medical Biology, Istanbul,Turkey; ${ }^{3}$ stanbul University, Cerrahpasa Medical Faculty, Department of Public Health, Istanbul, Turkey

10.1136/heartjnl-2011-300867.230

Introduction While numerous SNPs on chromosome 9p21 have been associated with coronary artery disease (CAD) in various populations, there are no reports of association on this locus in Turkish patients with existing CAD. We assessed the hypothesis that the $9 \mathrm{p} 21$ polymorphisms may be associated with increased risk and severity in patients with documented CAD.

Methods We genotyped 220 Turkish CAD patients and 240 Turkish disease-free controls by using real-time polymerase chain reaction (RT-PCR) to investigate the association between 2 SNPs, rs1333049 and rs2383207, on chromosome 9p21 and CAD risk and severity. Logistic regression was used to calculate the adjusted odds ratios (OR) and 95\% CI.

Results: In this study, there was a significant difference in the genotype distribution of both SNPs, rs1333049 and rs2383207, between CAD patients and controls $(\mathrm{p}<0.05)$ with OR of 1.81 (95\% CI 1.05 to 3.12 ) and 2.12 (95\% CI 1.19 to 4.10 ), respectively. After adjustment of CAD risk factors such as smoking, family history of $C A D$ and diabetes with multivariate logistic regression analysis, homozygous AA genotype increased CAD risk with an OR of 3.69 for rs 2383207 paradoxically with other reports conducted in different patient populations. However, rs1333049 had lost its significance on risk of CAD with an OR of 0.49 after adjustment of CAD risk factors. We also found a significant relation between rs1333049 and rs2383207 and GENSINI scores representing the severity of the CAD in our patient $(\mathrm{p}<0.001)$.

Conclusion This is the first study on the Turkish population showing an association between rs2383207 and rs1333049 polymorphisms on chromosome 9p21 and CAD. In conclusion, this study suggests that the polymorphisms rs 2383207 and rs1333049 on chromosome 9p21 are associated with the increased risk and the severity of CAD. However, further confirmation of these findings is needed. 